\title{
Metabolic changes during the menstrual cycle
}

\author{
BY J. T. BISDEE \\ Dunn Clinical Nutrition Centre, 100 Tennis Court Road, Cambridge CB2 IQL \\ AND P. J. GARLICK AND W. P. T. JAMES* \\ Rowett Research Institute, Bucksburn, Aberdeen AB2 9SB
}

(Received 13 June 1988 - Accepted 13 January 1989)

\begin{abstract}
1. Eight women maintained on a constant diet throughout a single menstrual cycle were studied for cyclical changes in body-weight, carbohydrate, fat and protein metabolism.

2. A cyclical variation in weight occurred with two phases corresponding to the time of ovulation and menstruation.

3. Creatinine and nitrogen excretion in the urine also tended to be higher in the luteal phase.

4. Analysis of gas exchange in a respiratory chamber for $24 \mathrm{~h}$ periods at intervals during the cycle suggested that carbohydrate utilization tended to be less in the early follicular phase.

5. Feeding with a mixed diet produced marked increases in carbohydrate utilization during the day with little change in fat utilization from the fasting state. Carbohydrate utilization was less when fasting at night and tended to be less when fed during the day in the early follicular phase of the menstrual cycle.

6. Weight changes were not readily ascribed to changes in colonic function, or in glycogen or body protein storage.
\end{abstract}

It has long been recognized that there are cyclical changes in body-weight and in water and electrolyte metabolism in the course of the menstrual cycle (Thorn et al. 1938; Danforth et al. 1946; Morton, 1950; Lamb et al. 1953; Morton et al. 1953; Thomas, 1953; Chesley \& Hellman, 1957; Fortin et al. 1958; Bruce \& Russell, 1962; Golub et al. 1965; Robinson \& Watson, 1965; Pliner \& Fleming, 1983). Glucose tolerance is also known to vary, it being proposed that insulin responsiveness is modified by sex hormones with fasting blood sugar levels above normal during menstruation, but with the lowest peak and the most rapid glucose disposal following the ingestion of a glucose load (Okey \& Robb, 1925).

Most earlier studies are complicated by the lack of control of food intake. Recently a series of studies have shown that energy intake may rise by $12-38 \%$ from the follicular to the luteal phase (Dalvit, 1981; Dalvit-McPhillips, 1983; Manocha et al. 1986), with a selective increase of about $50 \%$ in carbohydrate consumption, a feature known to affect electrolyte, glucose and nitrogen metabolism. More recently Calloway \& Kurzer (1982) have suggested that there are cyclical changes in $\mathbf{N}$ metabolism but in their study the food intake was maintained constant during each cycle.

In the present paper we summarize the effects of the menstrual cycle on body-weight, intestinal transit time, $\mathrm{N}$ balance and creatinine excretion in eight women previously studied for changes in total energy expenditure. In addition, analysis of the calorimetry findings in association with $\mathbf{N}$ excretion allowed an assessment of any changes in substrate oxidation induced by the hormonal changes.

\section{METHODS}

The overall protocol has been detailed in an earlier paper (Bisdee et al. 1989). The diet was designed as meat-free with $13 \%$ of energy as protein, $40 \%$ fat and $47 \%$ carbohydrate. The

* For reprints. 
intake of each meal was adjusted to provide one-third of the day's requirements, but no attempt was made to control or measure fluid or salt intake. Intakes were calculated to take account of the energy needs of each subject as they continued, as far as possible, with their usual activities throughout the study. After an initial calorimetric assessment, the intakes were adjusted in the hope of maintaining body-weight throughout the 1 month residential stay.

Body-weight was measured without clothes at the same time each day once the subjects had emptied their bladders. In analyzing cyclical changes, daily body-weights were first adjusted for any persistent trend over the whole of the residential period, and thereafter the mean quotient method (Danforth et al. 1946; Chesley \& Hellman, 1957; Watson \& Robinson, 1965) was used to assess changes relating to the stage of the cycle. This method involved finding the mean and standard deviation $(\sigma)$ of daily body-weights and then relating each daily deviation (d) from this mean weight, in terms of $\sigma$, to provide a mean weight quotient $(\mathrm{d} / \sigma)$ for each day. Body-weight changes were analyzed into eight parts of the menstrual cycle by dividing each of the four phases as described in the earlier paper (Bisdee et al. 1989), i.e. early and late follicular and luteal phases each divided into two. Weights were then assessed for differences in each part of the cycle by an analysis of variance.

The diets were given in association with capsules containing radio-opaque markers suitable for ready identification and counting after X-raying the frozen faecal samples (Branch \& Cummings, 1978). On the basis of changes in basal body temperature and the profiles of the preceding cycles monitored in the pre-residential phase of the study, the type of marker was changed each week at the estimated beginning of each of the four menstrual phases described in the earlier paper. Faecal samples containing more than $96 \%$ of one marker were pooled to provide a sample corresponding to each of the four phases, adjustments in estimating nutrient excretion then being made to take account of the marker content. The mean transit of the markers was calculated by the method described by Cummings et al. (1976). $\mathrm{N}$ balance was calculated after digesting duplicate freeze-dried samples of the diets, faecal samples and $24 \mathrm{~h}$ urinary samples for analysis of $\mathrm{N}$ using the Kjeldahl method. Assay variability in the Kjeldahl analysis calculated from duplicate samples proved to be $2.5 \%$. No attempt was made to include or exclude menstrual losses from the urinary samples obtained during menstruation. Creatinine was also measured in the daily urine samples by an autoanalyser technique based on the Jaffe reaction using the COBAS Bio System (Roche Products); the coefficient of variation of this method was $1.5 \%$.

Substrate utilization was calculated from the oxygen uptake and carbon dioxide production values using equations of Garlick (1987). Utilization calculated from gas exchange is the amount of substrate which is oxidized or converted to other metabolic products. During fasting this is taken from body stores: during feeding both diet and body stores may be utilized and total utilization is the sum of dietary intake plus the amount withdrawn from store. These calculations were applied to the $24 \mathrm{~h}$ values and also to fasting (midnight-06.00 hours) and the fed state between 09.30 and 21.30 hours. Urinary $N$ excretion $(24 \mathrm{~h})$ was used to calculate protein oxidation and, where appropriate, was proportioned into ratios for the fed and fasting states according to measurements made by Clugston \& Garlick (1982) of leucine oxidation rates at these times. This allowed each individual's $\mathrm{N}$ output to be used both in calculating nutrient utilization on a $24 \mathrm{~h}$ basis and during the fasting and fed periods. A $1 \%$ error in respiratory quotient measurement would change carbohydrate utilization by about $1 \mathrm{~g} / \mathrm{h}$ (Garlick et al. 1987).

Statistical analyses involved an analysis of variance in each comparison. 


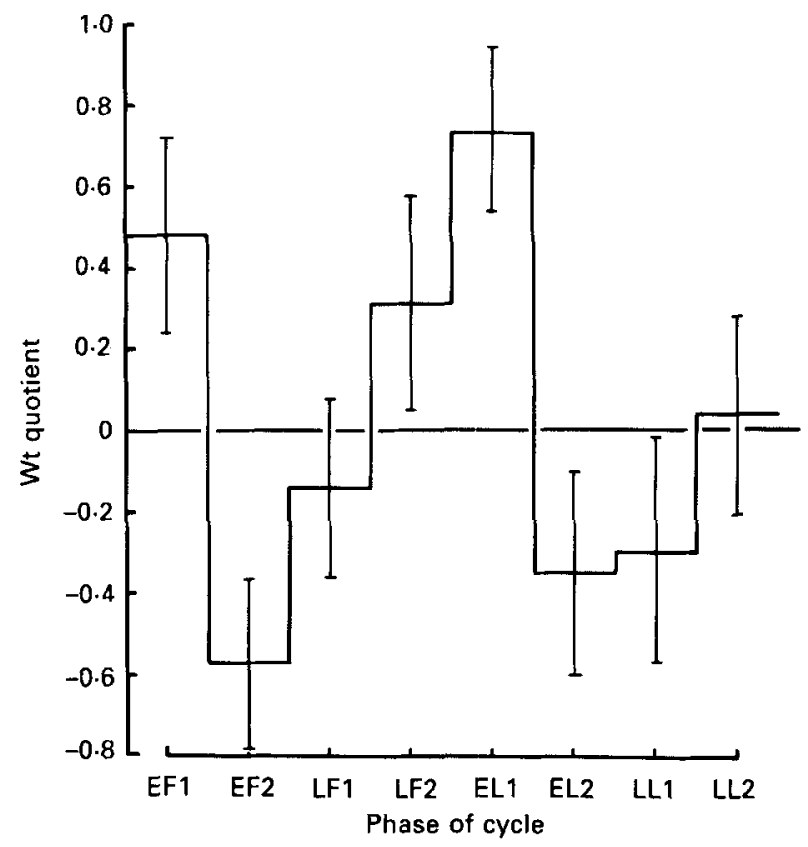

Fig. 1. Mean weight quotients for eight phases of the menstrual cycle. For details of weight quotient and division of the cycle into four phases, see p. 642. EF, early follicular; LF, late follicular; EL, early luteal; LL, late luteal phases. Values are means, with their standard errors represented by vertical bars, for eight subjects.

\section{RESULTS}

Despite adjusting the energy intake of the volunteers in the first few days of their residential stay, four of the eight lost weight at a significant rate amounting, on average, to $37 \mathrm{~g} / \mathrm{d}$. Two, however, were gaining at an average of $35 \mathrm{~g} / \mathrm{d}$ and the overall mean effect on the group amounted to a loss of only $10 \mathrm{~g}$ daily. Fig. 1 summarizes the changes in body-weight during the cycle, allowance having been made for long-term trends as described previously (p. 642). There were two peaks in body-weight, one during the early menstrual phase and the second corresponding to the time immediately after ovulation.

These peaks in body-weight while the subjects were on a constant diet could have resulted from changes in water balance or from alterations in colonic function, with changes in the sequestration of water and dietary residues in the colon. Water intake was not controlled, but Table 1 shows the collated values for urinary volumes and faecal weights in each of the eight periods of the cycle, corresponding to the weight changes depicted in Fig. 1. Complete faecal collections were obtained for all women as judged by marker output.

The daily faecal outputs were high, as was expected from the high fibre intakes of the group. For reasons discussed in the preceding paper, the experimental diet was meat-free and as such had a calculated dietary fibre content measured by the Southgate method (Southgate \& Durnin, 1970) of $42 \mathrm{~g} / \mathrm{d}$; this is approximately double that observed for the average meat-eating Cambridgeshire woman, but is typical of UK vegetarians (Bingham et al. 1979; Gear et al. 1979).

The mean transit time (h) did not differ in the four main phases of the cycle, being 43 (SE 14) and 40 (SE 10) in the early and late follicular phases and 41 (SE 10) and 45 (SE 13) in the early and late luteal phases. Similarly the marker-corrected measurements of faecal energy 




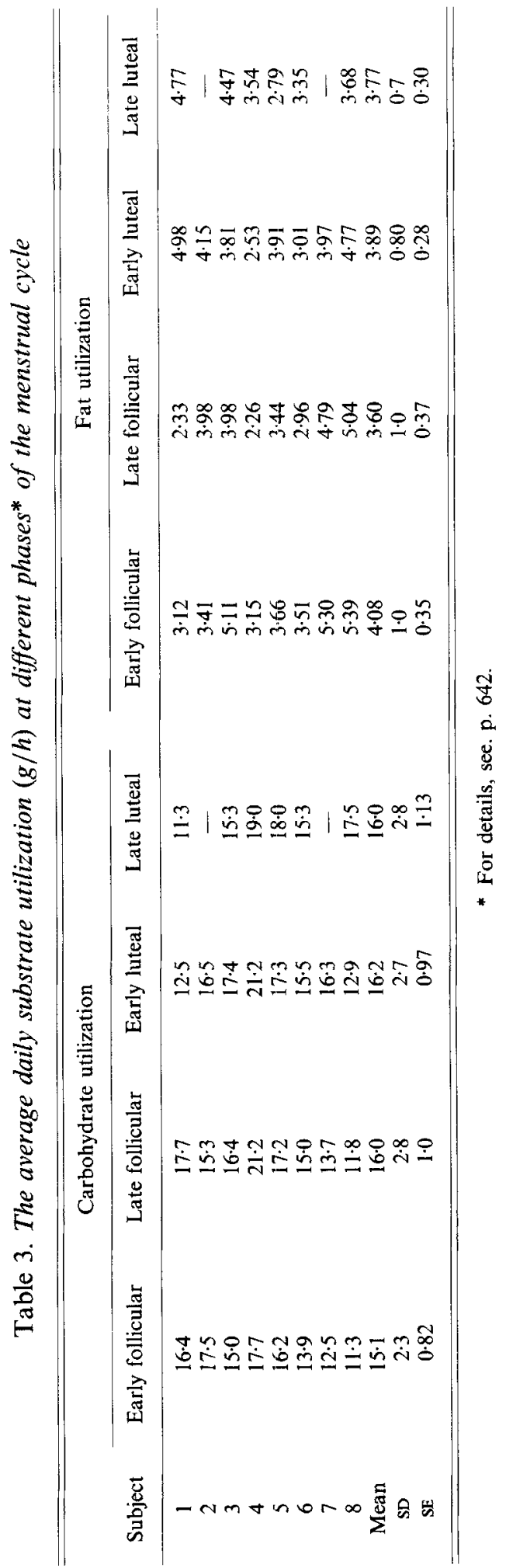



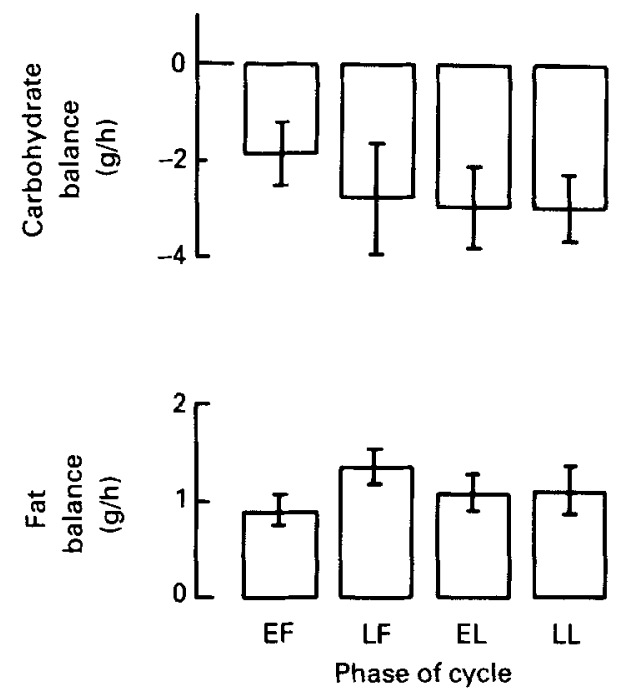

Fig. 2. Balance of carbohydrate (glycogen) and fat stores at four phases of the menstrual cycle for 24 $h$ periods in the calorimeter; for details of procedures, see p. 642. The follicular (F) and luteal (L) phases were divided into early $(\mathrm{E})$ and late $(\mathrm{L})$ parts. ANOVA shows that the differences between phases were not significant. Values are means, with their standard errors represented by vertical bars, for eight subjects (six subjects for the LL phase).

and faecal $\mathrm{N}$ did not differ during the four phases of the cycle (unpublished values). This implies that there were no significant changes in colonic motility or total energy or $\mathrm{N}$ handling during the menstrual cycle which could be ascribed to endocrinological influences.

Whether there was any change in renal function was somewhat less certain. The day-today variation in urinary output of both creatinine and $\mathrm{N}$ (Table 2) was small: withinsubject variability ranged from 7.9 to $16.1 \%$ for creatinine, and from 1.5 to $3.1 \%$ for urinary $\mathrm{N}$. The greater variation in daily creatinine excretion was expected as a result of the slight variation in daily creatinine intake; while the diet did not include meat, it did include some prawns on two of the three diet days. The day-to-day consistency in urinary $\mathrm{N}$ and creatinine output suggests that the urine collections were complete and that any cyclical trends in output reflect changes in renal function. Both urinary $\mathrm{N}$ and creatinine output were elevated during the luteal phase of the menstrual cycle by an average 4 and $5 \%$ respectively (Table 2). However, these differences were not significant and detailed examination of the daily values showed that there was no progressive change in $\mathrm{N}$ or creatinine output analogous to the observed changes in body-weight, shown in Fig. 1. Indeed, there was a rise in urinary $\mathrm{N}$ and therefore a fall in apparent $\mathrm{N}$ balance during the early luteal phase when the overall peak in body-weight occurred. Thus, there was no evidence of a fall in glomerular filtration or increase in $\mathrm{N}$ retention during the progressive weight increase observed from the early follicular to the early luteal phases shown in Fig. 1 .

An alternative explanation for cyclical changes in body-weight might be alterations in the amount of energy stored as glycogen at different times of the menstrual cycle. Table 3 shows carbohydrate and fat utilization rates as estimated by calorimetric methods for the whole $24 \mathrm{~h}$ period. Carbohydrate utilization was lower in the early follicular phase than at the other times, but the changes did not reach statistical significance. Total carbohydrate utilization was on average in excess of the carbohydrate intake while the subjects were in the calorimeters, so no carbohydrate storage as glycogen appeared to occur during the 
Fasting
Midnight-6.00 hours

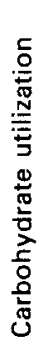

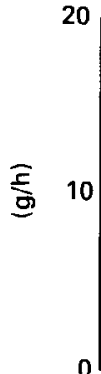

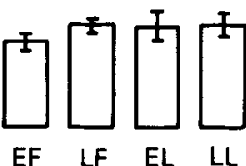

Phase of cycle

Fed

$09.30-21.30$ hours

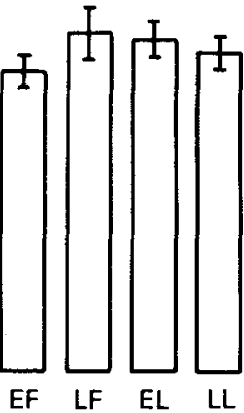

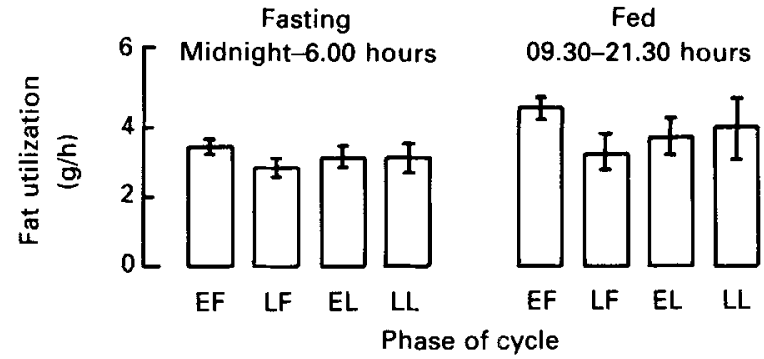

Fig. 3. Carbohydrate and fat utilization in four phases of the menstrual cycle from midnight to 06.00 hours while fasting and then during the day when fed. The follicular (F) and luteal (L) phases were divided into early (E) and late (L) parts. Values are means, with their standard errors represented by vertical bars, for eight subjects (six subjects for the LL phase). Differences between phases of the menstrual cycle did not reach statistical significance.

calorimeter period at any phase of the cycle. If carbohydrate utilization in excess of dietary intake can be equated with the metabolism of endogenous glycogen, i.e. negative carbohydrate balance (Fig. 2), then it was at a maximum in the late luteal phase, when it amounted to 72 (SE 19) g/d and at a minimum in the early follicular phase (44 (SE 24) g/d) (see Fig. 2), but this difference did not reach statistical significance. These two extremes corresponded to the two peaks in body-weight, which suggests that differences in glycogen storage during the cycle may not account for the peak in body-weight. Fig. 3 shows the rates of substrate utilization during the night-time, while fasting from midnight to 06.00 hours, and during the day while feeding. Again the women, while fasting, had a minimum rate of carbohydrate utilization during menstruation (i.e. early follicular phase) and a maximum rate 1 week later in the late follicular phase; the two values were not statistically different. Fat utilization during fasting was maximum in the early follicular phase and minimum in the late follicular phases. The striking increase in carbohydrate metabolism on feeding was evident, the rate of fat utilization being little different from the fasting state. Again, however, the rate of carbohydrate utilization was lowest in the first few days of the cycle and then rose to a maximum value before ovulation. 


\section{DISCUSSION}

This analysis of physiological changes during the menstrual cycle shows that even when food intake is carefully standardized there are some changes in body-weight and in carbohydrate, fat and $\mathrm{N}$ metabolism but not so great as to achieve statistical significance on an analysis of variance. The inter-individual differences were highly significant and the analysis displayed the problem of trying to discriminate small differences in metabolism in studies on energy balance. If food intake had been unlimited then the changes may have been much greater, but these would then have reflected the combined effects of altered nutrient intake and endogenous changes associated with the menstrual cycle. Wald et al. (1981) have suggested that small intestinal function might alter, because hydrogen breath tests after meals indicated that upper gastrointestinal transit time was significantly prolonged in the luteal phase of the cycle. The present findings of a constant mean transit time throughout the menstrual cycle presumably reflect the dominance of the colon in the overall transit of food through the gastrointestinal tract. In these eight volunteers there was no evidence of increased faecal weight, energy, solids or $\mathrm{N}$, nor was there a decreased transit time associated with any phase of the cycle.

The changes in creatinine excretion were unexpected, since Calloway \& Kurzer (1982) observed a cyclical pattern in only two of six subjects. Their subjects, however, showed a progressive decline in creatinine output while on a creatine-free diet which may have complicated their analysis. Six of eight subjects in the present study showed an increase in creatinine output in the luteal phase (Table 2), but the difference between the phases was not significant. Calloway \& Kurzer (1982) observed two cycles in urinary N, with the lowest output at the time of ovulation, with two peaks in the mid-follicular and mid-luteal phases. Given the design of our study, where the cycle was deliberately divided into four phases for the purpose of calorimetry, a corresponding analysis of $\mathrm{N}$ and creatinine output was used. Analysis of daily $\mathrm{N}$ and creatinine output in a manner analogous to that of Calloway \& Kurzer (1982) showed that there seemed to be a cyclical pattern of $\mathrm{N}$ and creatinine output, but this did not match the weight changes displayed in Fig. 1. Analyses of water and sodium balance were not made in our study so changes in electrolyte storage are unclear.

Although the changes in creatinine excretion might reflect changes in renal function, given the similar changes in urinary $\mathrm{N}$ output, a further plausible explanation is that the elevated basal temperature might be responsible for the increased creatinine output. Creatinine is generated by the physical and spontaneous cyclization of creatine phosphate in muscle, so an increase in basal body temperature of about $0.5^{\circ}$ (the value found) would, on a $Q_{10}=2$ basis (a rise in temperature of $10^{\circ}$ resulting in a doubling of the rate of reaction) therefore, be expected to increase creatinine output by about $5 \%$ in the luteal phase. A change of this magnitude was observed.

The changes in substrate utilization during the menstrual cycle have not been documented previously, but it is recognized that metabolic responses to insulin may be modified by oestrogens and progesterone. Okey \& Robb (1925), in an extensive analysis of cyclical changes in fasting blood glucose and in oral glucose tolerance, found that although fasting plasma glucose tended to be higher at the time of menstruation, the peak plasma concentration was reduced and a more pronounced hypoglycaemia occurred after $2 \mathrm{~h}$. This is consistent with greater insulin responsiveness to food at the time of menstruation. This would perhaps accord with the tendency to utilize less and store more carbohydrate during feeding in the early follicular phase (Fig. 3).

The calorimeter measurements tended to be conducted in the later part of each of the four phases and should most realistically be related, therefore, to the second component of each phase of body-weight changes shown in Fig. 1. On this basis it is difficult to see any 
relations between the peaks and troughs in both weight and the rates of carbohydrate utilization.

These results also illustrate the dominance of carbohydrate metabolism as individuals swing from the fasted to the fed state. This point has recently been noted by Flatt et al. (1985) and Garlick et al. (1987), and is highlighted in the results shown in Fig. 3. It also implies that the normal route of metabolism for fat allows ready sequestration of dietary fat into stores rather than oxidation. Whether this sequestration of fat is more readily achieved during one phase of the cycle is difficult to substantiate, but fat utilization does appear to be at its lowest in the early luteal phase of the cycle (Table 3 ). The values given for fat metabolism are, of course, net values and do not include fatty acid oxidation, which is balanced by fat synthesized from dietary carbohydrate. Thus, to obtain a more complete picture of what is actually happening, one needs to distinguish the direct oxidation of glucose from its conversion to fat.

The authors thank Dr Ann Ralph for her help with the calculations and Mrs Hazel Vint for statistical advice.

\section{REFERENCES}

Bingham, S., Cummings, J.H. \& McNeil, N. I. (1979). Intakes and sources of dietary fibre in the British population. American Journal of Clinical Nutrition 32, 1314-1319.

Bisdee, J. T., James, W. P. T. \& Shaw, M. A. (1989). Changes in energy expenditure during the menstrual cycle. British Journal of Nutrition 61, 187-199.

Branch, W. J. \& Cummings, J. H. (1978). Comparison of radio-opaque pellets and chromium sesquioxide as inert markers in studies requiring faecal collections. Gul 19, 371-376.

Bruce, J. \& Russell, G. F. M. (1962). Premenstrual tension: a study of weight changes and balances of water, sodium and potassium. Lancet ii, 267-271.

Calloway, D. H. \& Kurzer, M. S. (1982). Menstrual cycle and protein requirements of women. Journal of Nutrition 112, 356-366.

Chesley, L. C. \& Hellman, L. M. (1957). Variations in body weight and salivary sodium in the menstrual cycle. American Journal of Obstetrics \& Gynecology 74, 582-590.

Clugston, G. A. \& Garlick, P. J. (1982). The response of protein and energy metabolism to food intake in lean and obese man. Human Nutrition: Clinical Nutrition 36C, 57-70.

Cummings, J. H., Jenkins, D. J. A. \& Wiggins, H. S. (1976). Measurement of the mean transit time of dietary residue through the human gut. Gut $17,210-218$.

Dalvit, S. P. (1981). The effect of the menstrual cycle on patterns of food intake. American Journal of Clinical Nutrition 34, 1811-1815.

Dalvit-McPhillips, S. P. (1983). The effect of the human menstrual cycle on nutrient intake. Physiology and Behaviour 31, $209-212$.

Danforth, D. N., Boyer, P. K. \& Graff, S. (1946). Fluctuations in weight, haematocrit and plasma protein with the menstrual cycle. Endocrinology 39, 188-193.

Flatt, J. P., Ravussin, E, Acheson, K. J. \& Jequier, E. (1985). Effects of dietary fat on postprandial substrate oxidation and on carbohydrate and fat balances. Journal of Clinical Investigation 76, 1019-1024.

Fortin, J. N., Wittkower, E. D. \& Kalz, F. (1958). A psychosomatic approach to the pre-menstrual tension syndrome: a preliminary report. Canadian Medical Association Journal 79, 978-98I.

Garlick, P. J. (1987). An evaluation of the formulae for calculating nutrient oxidation rates from respiratory gas measurements. Human Nutrition: Clinical Nutrition 41C, 165-176.

Garlick, P. J., McHardy, K. C., McNurlan, M. A., Calder, A. G., Milne, E., Fearns, L. M. \& Broom, J. (1987). Rates of nutrient utilization in man measured by combined respiratory gas analysis and stable isotopic labelling: the effect of food intake. Human Nutrition: Clinical Nutrition 41C, 177-191.

Gear, J. S. S., Fursdon, P., Nolan, D. J., Ware, A., Mann, J. I., Brodribb, A. J. M. \& Vessey, M. P. (1979). Symptomless diverticular disease and intake of dietary fibre. Lancet i, 511-514.

Golub, L. J, Menduke, H. \& Conly, S. S. (1965). Weight changes in college women during the menstrual cycle. American Journal of Obstetrics and Gynecology 91, 89-94.

Lamb, W. M., Ulett, G. A., Masters, W. H. \& Robinson, D. W. (1953). Premenstrual tension: EEG, hormonal and psychiatric evaluation. American Journal of Psychiatry 102, 840-846.

Manocha, S., Choudhuri, G. \& Tandon, B. N. (1986). A study of dietary intake in pre- and post-menstrual period. Human Nutrition: Applied Nutrition 40A, 213-216.

Morton, J. H. (1950). Premenstrual tension. American Journal of Obstetrics and Gynecology 60, $343-352$. 
Morton, J. H., Additon, H., Addison, R. G., Hunt, L. \& Sullivan, J. J. (1953). A clinical study of premenstrual tension. American Journal of Obstetrics and Gynecology 65, 1182-1191.

Okey, R. \& Robb, E. I. (1925). Studies of the metabolism of women. 1. Variations in the fasting blood sugar level and in sugar tolerance in relation to the menstrual cycle. Journal of Biological Chemistry 65, 165-186.

Pliner, P. \& Fleming, A. S. (1983). Food intake, body weight, and sweetness preferences over the menstrual cycle in humans. Physiology and Behaviour 30, 663-666.

Robinson, M. F. \& Watson, P. E. (1965). Day to day variations in body weight of young women. British Journal of Nutrition 19, 225-235.

Southgate, D. A. T. \& Durnin, J. V. G. A. (1970). Calorie conversion factors: an experimental reassessment of the factors used in the calculation of the energy value of human diets. British Journal of Nutrition 24, 517-535.

Thomas, C. B. (1953). Some observations on the relationship between weight changes following sodium restriction and those associated with the menstrual cycle in young women. Annals of Internal Medicine 39, $289-305$.

Thorn, G. W., Nelson, K. R. \& Thorn, D. W. (1938). A study of the mechanism of edema associated with menstruation. Endocrinology 22, 155-163.

Wald, A., Van Thiel, D. H., Hoechsetter, L., Gavaler, J. S., Egler, K. M., Verm, R., Scott, L. \& Lester, R. (1981). Gastrointestinal transit: the effect of the menstrual cycle. Gastroenterology 80, 1497-1500.

Watson, P. E. \& Robinson, M. F. (1965). Variations in body weight of young women during the menstrual cycle. British Journal of Nutrition 19, 237-248. 